\title{
Liver Function and Anemia Pathogenesis in Iranian Traditional Medicine
}

\author{
Saeedeh Yarjou ${ }^{1}$; Omid Sadeghpour ${ }^{2}$; Esmail Nazem ${ }^{3}$; Amir Hossein Emami ${ }^{4, *}$ \\ ${ }^{1}$ Department of Traditional Medicine, Tehran University of Medical Sciences, Tehran, IR Iran \\ $2^{2}$ Department of Herbal Medicine, Research Institute for Islamic and Complementary Medicine, Iran University of Medical Sciences, Tehran, IR Iran \\ 3 Faculty of Traditional Medicine, Tehran University of Medical Sciences, Tehran, IR Iran \\ ${ }^{4}$ Department of Hematology/Oncology, Imam Khomeini Hospital, Tehran University of Medical Sciences, Tehran, IR Iran \\ ${ }^{*}$ Corresponding Author: Amir Hossein Emami, Department of Hematology Oncology, Imam Khomeini Hospital, Tehran University of Medical Sciences, Tehran, IR Iran. Tel: +98- \\ 2166581593, Fax: +98-2166581593, E-mail:emamiami@yahoo.com \\ Received: December 24, 2013; Revised: April 29, 2014; Accepted: June 16, 2014
}

\begin{abstract}
Background: Nutritional deficiency, bleeding, and inflammation are three main causes of anemia. On the other hand, erythropoietin (EPO) production, iron availability, and a healthy bone marrow are essential for erythropoiesis. Recently, recombinant human erythropoietin (rhEPO) has been used to treat the patients already taking iron supplements. In Iranian traditional medicine literature, much has been written about anemia and its treatment.

Objectives: This study aimed to review Iranian traditional medicine theories in the treatment of anemia.

Materials and Methods: In the present study, authors have attempted to find the etiology and pathogenesis of anemia in Iranian traditional Medicine Literature and the views of ancient physicians in this field.

Results: Our findings proved that Iranian traditional medicine defined anemia as blood humor production dysfunction, which is caused by several reasons mainly hepatic dysfunction. Thus, liver is supposed to be the most important organ in this regard. This will make considering hepatoprotective medicines in anemia even in patients with non-hepatic problems.

Conclusions: The article presents the relation between anemia and liver dysfunction viewed by Iranian traditional medicine as an important finding in designing a treatment plan. Despite the new findings on the evaluation of liver function (i.e. laboratory tests), it seems that further studies for earlier evaluation of liver function in anemia are highly required.
\end{abstract}

Keywords:Anemia; Traditional Medicine; Liver Diseases

\section{Background}

Oxygen is essential for tissue metabolism and also affects the production of erythropoietin in kidneys, which induce red blood cell production in bone marrow. This confirms the key role of oxygen in hematopoiesis regulation (1). About $90 \%$ of anemic cases are caused by three main reasons: nutritional deficiencies, chronic disease, and bleedings (2). Production of erythropoietin (EPO), adequate iron, and a healthy bone marrow are essential factors for erythropoiesis (3). Recently, erythropoietic agents (as effective nephroprotectives) are being used to treat anemia caused by chronic inflammatory disorders. Also intravenous iron supplements are added to maximize the effect $(1,4)$. Iranian traditional medicine scholars and physicians described anemia as the blood humor (a moist and fluid substance into which aliment is first transformed and it is hot and moist by nature) deficiency and call it Qillat-o-dam $(5,6)$.

\subsection{Blood and Blood Humor}

The mechanism of humor production in the liver is briefly presented as follows. When food enters into the stomach, it is converted to a substance called Chylos (a fluid substance resembling thick barely water, result of the food digestion for the first time and it is different from the chylos, which passes through the lymphatic veins), which is then ready to be delivered into the liver $(5,7)$. Chylos tenuity is absorbed in the liver by mesenteric vessels, then it maturates in the liver and becomes humors $(5,7)$. Generally, liver is responsible for humors production, especially blood humors, to feed the whole body $(5,7)$. This shows the critical function of liver in blood humor formation. Likewise, Jorjani (1042-1137 AD) mentioned in his book, Zakhireh Kharazmshahi: "Liver is the principal organ or source of physical faculties" (5). There are three factors affecting the blood humor production by liver: 1 ) hepatic equable heat (normal hepatic temperament), 2) proper and equable food and beverages from temperamental point of view, and 3) adequate maturation of Chylos by stomach. These will make the body being fed and kept warm and moist $(5,7)$. Body humors imbalance were diagnosed by tradintional physicians through inspecting patient's blood samples and determining the quality and ratio of each humor there.

\section{Objectives}

This study aimed to evaluate Iranian traditional medicine theories in the treatment of anemia. 


\section{Materials and Methods}

In this study, the authors reviewed the Iranian traditional medicine literature and after comparing the relevant texts, the pathogenesis and etiology of anemia were discussed.

\section{Results}

From the perspective of Iranian traditional medicine, anemia is directly related to the liver and physical faculties.

\subsection{Anemia Pathophysiology}

Physical faculties (which might nourish the body until the end of life and hasten its growth to its maximum potential) play a key role in humors production, especially blood humor. Liver as the source of this faculty converts the nutrients into the resembled intended organ by nutritive faculty, (the faculty, which changes the nutriment into the likeness of the nourished organ and makes up for the loss) a branch of physical faculty (7). This process is damaged in three ways. First, conversion of food to humors, mainly blood, which is finely produced in the liver $(5,7)$ can be interrupted by three factors: 1 ) gastric digestive faculty dysfunction, 2) hepatic digestive faculty dysfunction, and 3) and absorptive faculty (attracts what is beneficial i.e. nutriment) disorder, which leads to the malnutrition in the organ (called atrophia) $(5,7)$. It is obvious that liver and stomach digestive faculties function for the whole body.

Second, when the nutrient changes into an organ component. Digestive and expulsive faculties' dysfunction (expulsive faculties, which expels the superfluous matter through the natural passages of orifices) interferes with nutrients becoming an organ's component, as in anasarca $(5,7)$.

Third, when the nutrient becomes similar to the organ in all aspects, even in texture and color. Sometimes it is interfered with, as in vitiligo and leukoderma, when digestive faculty is defected or not functioning properly. This causes the presence of acquisition and adhesion without any resemblance $(5,7)$. In conclusion, according to the Iranian traditional medicine, physical faculty's condition is the most important factor in anemia pathogenesis. However, some of the above disorders like vitiligo, leukoderma, anasarca, and atrophia are not classified under anemia etiology.

\subsection{Anemia Etiology}

Based on Iranian traditional medicine observations, the basic mechanism of anemia is nutritive faculty dysfunction $(5,7)$. Therefore, anemia could be associated with both endogenous and exogenous factors classified below.

\subsubsection{Food}

Eating disorders may induce anemia based on their quality and quantity aspects. Malnutrition or taking unsuitable foods for blood humor may cause anemia $(5,6,8)$.

\subsubsection{Drugs and Toxins}

Some drugs and toxins may cause body temperament to become cool or desiccated. This status prevents blood humor production and may cause anemia $(5,6,8)$.

\subsubsection{Blood Loss}

Sometimes anemia is caused by bleedings such as menorrhagia, hemorrhoids bleedings, wet cupping, dysentery, etc. $(5,6,8)$.

\subsubsection{Psychological Factors}

Depression, horror and the like may interfere with blood humor production through nutritive faculty.

\subsubsection{Gastric Disorders}

Gastric disorders such as gastric intemperaments, inflammations, and digestive disorders may lead to disturb chylos production. This will make liver incapable of producing excellent blood humor and so anemia occurs (5-7).

\subsubsection{Hepatic Diseases}

Hepatic disorders are classified into two groups.

\subsubsection{Hepatic Primary Diseases}

Hepatic disorders such as intemperaments, inflammations, obstructions (Sodad), as well as liver asthenia can lead to hepatic dysfunction. Then anemia will happen as a result of imperfect blood humor production $(5,6)$.

\subsubsection{Hepatic Secondary Diseases}

Many different factors influence a healthy liver; one of the most important factors is proper functioning of related organs.

Stomach, kidney, spleen, and gall bladder disorders affect the liver and may cause the most common secondary hepatic diseases, which lead to anemia. For instance, Jorjani mentioned in his book, Zakhireh Kharazmshahi: "people who suffers from congenital spleen enlargement, are susceptible to liver asthenia, which follows by ascites" $(5,6)$.

Uterus, bowel, lung, and the like may interfere with the liver function but with less significance. For example, severe menstruation absorbs the blood from the liver and makes its temperament cold. On the other hand, amenorrhea may lead to abnormal blood humor production in the liver $(5,6)$.

\section{Discussion}

Anemia along with liver diseases such as cirrhosis and chronic liver diseases, especially in association with renal dysfunction, enhances erythropoietin production $(9,10)$ and there is an inverse correlation between erythropoietin and hemoglobin level in chronic hepatic patients (11). But in this situation, erythropoietin level elevation is 
lower than what observed in iron-deficiency anemia and seems to be inadequate to compensate (10).

Currently, recombinant human erythropoietin (rhEPO) is used as a substitute therapy in patients with renal diseases, chronic and iron-deficiency anemia $(1,4,12)$. Considering the role of EPO in supporting liver regeneration after hepatectomy, its involvement in the regulation of angiogenesis and also the effect of rhEPO in reducing liver injury induced by ischemic reperfusion, this hormone seems to have an effective role in liver protection (13-15). There are documents on silymarin effectiveness in anemia as a hepatoprotective medicine, which emphasizes the importance of hepatic support in anemia (16). Also hepcidin made by hepatocytes blocks cellular iron exportation and is effective in mediating inflammation derived anemia $(17,18)$. According to Iranian traditional medicine, many pathogenic factors are involved in anemia. The most important function in this regard belongs to the liver as a vital organ for both blood humor production and its regulation.

Accordingly, using hepatoprotective medicines are essential in treating anemic patients even if there are no signs or symptoms of hepatic diseases. In other words, treating the liver as the producer of blood humor has to be prioritized in these patients in order to correct anemia. Then, observing other related organs such as stomach, spleen, and gall bladder and treating their disorders is also important. Although there are some reports that aspartate transaminasis to platelet ratio index (APRI) score can be used as a non-invasive marker in liver fibrosis $(19,20)$, there might be no compulsion in changing APRI in the process involved liver dysfunction in anemia.

Citing from Ibn-Sina's previous study about anemia, he described the pathophysiology and treatment of many pathological conditions related to anemia such as ascites, digestive dysfunction, hepatitis, and so on. He also indicated the different causes for hepatic dysfunction in a logical sequence, which seems to be a very specific scientific classification no less different from what we use nowadays. He also mentioned all the organs that contribute to liver asthenia, which lead to anemia.

This article presents the relation between anemia and liver dysfunction from Ibn-Sina's viewpoint, which was an important element in designing a treatment plan. Although there are new findings about the evaluation of liver function in diseases, further studies for earlier evaluation of liver function in anemia is highly recommended.

\section{Acknowledgements}

The authors are grateful to authors, editors, and publishers of all books, journals, and articles from where the subject of this study has been reviewed and discussed. Also they are grateful to Dr Hamid Namavar for editing the article.

\section{Authors' Contributions}

Study concept and design: Amir Hossein Emami, Saeedeh Yarjou, and Omid Sadeghpour; drafting the manuscript: Saeedeh Yarjou; critical revision of the manuscript for important intellectual content: Esmail Nazem, Omid Sadeghpour, and Amir Hossein Emami.

\section{References}

1. Moore E, Bellomo R. Erythropoietin (EPO) in acute kidney injury. Annals of Intensive Care. 2011;1(1):3.

2. Moreno Chulilla JA, Romero Colas MS, Gutierrez Martin M. Classification of anemia for gastroenterologists. World J Gastroenterol. 2009;15(37):4627-37.

3. Wiener C, Fauci A, Braunwald E, Kasper D, Hauser S, Longo D, et al. Harrisons Principles of Internal Medicine.New York: McGrawHill Education; 2012.

4. Gasche C, Berstad A, Befrits R, Beglinger C, Dignass A, Erichsen $\mathrm{K}$, et al. Guidelines on the diagnosis and management of iron deficiency and anemia in inflammatory bowel diseases. Inflamm Bowel Dis. 2007;13(12):1545-53.

5. Jorjani SE. Zakhireh Kharazmshahi.Tehran: Amir kabir; 2012.

6. Azam M. Research Institute for Islamic and Complementary Medicine.Tehran: Exir Azam; 1387.

7. Avicenna H. Al-Qanun fi'l-Tibb (Canon of Medicine).New Delhi: Jamia Hamdard; 1993.

8. Garshi AA,. Explanation of Canon, Handwritten Manuscript.Tehran Research Institute for Islamic and Complementary Medicine library.

9. Jensen JD, Jensen LW, Madsen JK, Poulsen L. The metabolism of erythropoietin in liver cirrhosis patients compared with healthy volunteers. Eur J Haematol. 1995;54(2):111-6.

10. Bruno CM, Neri S, Sciacca C, Bertino G, Di Prima P, Cilio D, et al. Plasma erythropoietin levels in anaemic and non-anaemic patients with chronic liver diseases. World J Gastroenterol. 2004;10(9):1353-6.

11. Tacke F, Luedde T, Manns MP, Trautwein C. Regulation of plasma erythropoietin in chronic liver disease. World J Gastroenterol. 2004;10(19):2922-3.

12. Madro A, Kurzepa J, Czechowska G, Slomka M, Celinski K, Szymonik-Lesiuk S. Erythropoietin inhibits liver gelatinases during galactosamine-induced hepatic damage in rats. Pharmacol Rep. 2009;61(5):917-23.

13. Greif F, Ben-Ari Z, Taya R, Pappo O, Kurtzwald E, Cheporko Y, et al. Dual effect of erythropoietin on liver protection and regeneration after subtotal hepatectomy in rats. Liver Transpl. 2010;16(5):631-8.

14. Ribatti D, Vacca A, Roccaro AM, Crivellato E, Presta M. Erythropoietin as an angiogenic factor. Eur J Clin Invest. 2003;33(10):891-6.

15. Sepodes B, Maio R, Pinto R, Sharples E, Oliveira P, McDonald M, et al. Recombinant human erythropoietin protects the liver from hepatic ischemia-reperfusion injury in the rat. Transpl Int. 2006;19(11):919-26.

16. Karimi G, Vahabzadeh M, Lari P, Rashedinia M, Moshiri M. "Silymarin", a promising pharmacological agent for treatment of diseases. Iran J Basic Med Sci. 2011;14(4):308-17.

17. Ganz T. Hepcidin, a key regulator of iron metabolism and mediator of anemia of inflammation. Blood. 2003;102(3):783-8.

18. Weiss G, Gasche C. Pathogenesis and treatment of anemia in inflammatory bowel disease. Haematologica. 2010;95(2):175-8.

19. Parsian $\mathrm{H}$. Is the aspartate transaminase to platelet ratio index adequate for the assessment of liver fibrosis in patients with chronic liver disease? Hepat Mon. 2011;11(5):380-1.

20. Leonardi S, Vitaliti G. Liver fibrosis in patients with chronic liver diseases: are laboratory tests useful to diagnosis? Hepat Mon. 2011;11(5):382-3. 